\title{
Vector bundles and regulous maps
}

\author{
Marcin Bilski • Wojciech Kucharz • \\ Anna Valette - Guillaume Valette
}

Received: 24 July 2012 / Accepted: 25 November 2012 / Published online: 17 January 2013

(C) The Author(s) 2013. This article is published with open access at Springerlink.com

\begin{abstract}
Let $X$ be a compact nonsingular affine real algebraic variety. We prove that every pre-algebraic vector bundle on $X$ becomes algebraic after finitely many blowing ups. Using this theorem, we then prove that the Stiefel-Whitney classes of any pre-algebraic $\mathbb{R}$-vector bundle on $X$ are algebraic. We also derive that the Chern classes of any pre-algebraic $\mathbb{C}$-vector bundles and the Pontryagin classes of any pre-algebraic $\mathbb{R}$-vector bundle are blow$\mathbb{C}$-algebraic. We also provide several results on line bundles on $X$.
\end{abstract}

Keywords Real algebraic variety · Pre-algebraic vector bundle · Algebraic vector bundle . Multiblowup · Regulous map.

Mathematics Subject Classification (2000) $\quad$ 14P05 - 14P25 · $14 \mathrm{P} 99$.

Research partially supported by NCN grants 2011/01/B/ST1/01289, 2011/01/B/ST1/03875.

M. Bilski · W. Kucharz $(\varangle) \cdot$ A. Valette

Wydział Matematyki i Informatyki Uniwersytetu Jagiellońskiego, ul. S. Łojasiewicza 6, 30-348 Kraków, Poland

e-mail: wojciech.kucharz@im.uj.edu.pl

M. Bilski

e-mail: marcin.bilski@im.uj.edu.pl

A. Valette

e-mail: anna.valette@im.uj.edu.pl

G. Valette

Instytut Matematyczny PAN, ul. Św. Tomasza 30,

31-027 Kraków, Poland

e-mail: gvalette@impan.pl 


\section{Introduction}

The language of real algebraic geometry, as in [5], is used throughout this paper. Thus, an affine real algebraic variety is a locally ringed space isomorphic to an algebraic subset of $\mathbb{R}^{n}$, for some $n$, endowed with the Zariski topology and the sheaf of real-valued regular functions. A real algebraic variety is a locally ringed space that can be covered by finitely many open sets, each of which, together with the restriction of the structure sheaf, is an affine real algebraic variety, cf. [5, Definition 3.2.11]. The underlying topology of any real algebraic variety is called the Zariski topology. Each quasi-projective real algebraic variety is affine, cf. [5, Proposition 3.2.10, Theorem 3.4.4]. Morphisms of real algebraic varieties are called regular maps. Each real algebraic variety carries also the Euclidean topology, which is induced by the usual metric on $\mathbb{R}$. Unless explicitly stated otherwise, all topological notions relatin! $g$ to real algebraic varieties refer to the Euclidean topology.

Let $\mathbb{F}$ stand for $\mathbb{R}, \mathbb{C}$ or $\mathbb{H}$ (the quaternions). Only left $\mathbb{F}$-vector spaces will be considered. When convenient, $\mathbb{F}$ will be identified with $\mathbb{R}^{d(\mathbb{F})}$, where $d(\mathbb{F})=\operatorname{dim}_{\mathbb{R}} \mathbb{F}$.

Let $X$ be an affine real algebraic variety. In the present paper investigated are relationships between pre-algebraic and algebraic $\mathbb{F}$-vector bundles on $X$, cf. [5, Chapter 12] for the definitions of such bundles. It should be mentioned that pre-algebraic vector bundles and algebraic vector bundles had been called algebraic vector bundles and strongly algebraic vector bundles, respectively, in the literature predating the publication of [5], cf. for example $[2-4,7,11,16,17,22]$. A pre-algebraic $\mathbb{F}$-vector bundle on $X$ is an algebraic $\mathbb{F}$-vector bundle if and only if it is generated by global algebraic sections, cf. [5, Theorem 12.1.7]. By definition, the total space of a pre-algebraic $\mathbb{F}$-vector bundle on $X$ is a real algebraic variety which may not be affine; actually it is affine if and only if the bundle is algebraic, cf. [16,17]. An example of a pre-algebraic $\mathbb{R}$-line bundle on $\mathbb{R}^{2}$ that is not algebraic can be ! found in [2] or [5, Example 12.1.5]. Due to this, and other similar examples, pre-algebraic vector bundles have been viewed as pathological objects and almost completely neglected. They appear in the literature essentially as a preliminary step in the definition of algebraic vector bundles, cf. $[2-5,7,16,17,22]$. A single exception is [11], which contains a proof that any pre-algebraic vector bundle on a nonsingular variety is a Nash vector bundle (terminology as in [5]). However, pre-algebraic vector bundles do have other interesting properties.

In this paper it is proved that each pre-algebraic $\mathbb{F}$-vector bundle on $X$ can be made algebraic by successively blowing up $X$. A multiblowup of $X$ is a regular birational map $\pi: X^{\prime} \rightarrow X$ which is the composition of a finite number of blowups with nonsingular centers. In particular, $X^{\prime}$ is an affine real algebraic variety. Furthermore, if $X$ is nonsingular, then so is $X^{\prime}$.

Theorem 1.1 Let $\xi$ be a pre-algebraic $\mathbb{F}$-vector bundle on an affine real algebraic variety $X$. Then there exists a multiblowup $\pi: X^{\prime} \rightarrow X$ of $X$ such that the variety $X^{\prime}$ is nonsingular and the pullback $\mathbb{F}$-vector bundle $\pi^{*} \xi$ on $X^{\prime}$ is algebraic.

The proof is given in Sect. 2 .

Corollary 1.2 With notation as in Theorem 1.1, if the variety $X$ is nonsingular, then there exists a Zariski closed subset $Z$ of $X$ such that $\operatorname{codim}_{X} Z \geq 2$ and the restriction $\xi_{\mid X \backslash Z}$ is an algebraic $\mathbb{F}$-vector bundle on $X \backslash Z$.

Proof Let $\pi: X^{\prime} \rightarrow X$ be as in Theorem 1.1. Since the variety $X$ is nonsingular, there exists a Zariski closed subset $Z$ of $X$ of codimension at least 2 such that $\pi$ is a biregular isomorphism from $X^{\prime} \backslash \pi^{-1}(Z)$ onto $X \backslash Z$. This implies the assertion in Corollary 1.2. 
A completely different proof of Corollary 1.2 can be found in [2,22] (in [22] some problematic points of [2] are corrected).

Corollary 1.2 immediately implies the following:

Corollary 1.3 If $X$ is a nonsingular affine real algebraic variety of dimension 1, then every pre-algebraic $\mathbb{F}$-vector bundle on $X$ is algebraic.

In the subsequent sections, Theorem 1.1 is used to clarify to what extent the known results on characteristic classes of algebraic vector bundles can be carried over to pre-algebraic vector bundles. It turns out that such a generalization is possible for the Stiefel-Whitney classes (Theorem 3.2), while for the Chern and Pontryagin classes some new phenomena come into play (Sect. 5).

Pre-algebraic and algebraic vector bundles can be regarded as topological vector bundles. This is explored in the remainder of the present section.

Let $\mathbb{S}^{1}$ be the unit circle,

$$
\mathbb{S}^{1}=\left\{\left(x_{1}, x_{2}\right) \in \mathbb{R}^{2} \mid x_{1}^{2}+x_{2}^{2}=1\right\},
$$

and let $\mathbb{T}^{n}=\mathbb{S}^{1} \times \cdots \times \mathbb{S}^{1}$ be the $n$-fold product.

Proposition 1.4 For any integer $n>d(\mathbb{F})$, there exist a nonsingular affine real algebraic variety $X$ and a topological $\mathbb{F}$-line bundle $\xi$ on $X$ such that $X$ is diffeomorphic to $\mathbb{T}^{n}$ and $\xi$ is not isomorphic to any pre-algebraic $\mathbb{F}$-line bundle on $X$.

The proof is given in Sect. 3. In Proposition 1.4 the assumption $n>d(\mathbb{F})$ is essential.

Proposition 1.5 Let $X$ be a compact nonsingular affine real algebraic variety. If $\operatorname{dim} X=$ $d(\mathbb{F})$, then every topological $\mathbb{F}$-line bundle on $X$ is isomorphic to a pre-algebraic $\mathbb{F}$-line bundle.

Note that under the assumptions as in Proposition 1.5, there exists a nontrivial topological $\mathbb{F}$-line bundle on $X$. Proposition 1.5 is well known if $\mathbb{F}=\mathbb{R}$, cf. [5, Theorem 12.5.1]. The proof for $\mathbb{F}=\mathbb{C}$ and $\mathbb{F}=\mathbb{H}$ is given in Sect. 4 . It is worthwhile to contrast Proposition 1.5 (for $\mathbb{F}=\mathbb{C}$ or $\mathbb{F}=\mathbb{H}$ ) with the behavior of algebraic vector bundles.

Example 1.6 Every algebraic $\mathbb{C}$-vector bundle on $\mathbb{T}^{n}$ is algebraically stably trivial, cf. [6] or [5, Corollary 12.6.6]. Consequently, every algebraic $\mathbb{C}$-line bundle on $\mathbb{T}^{n}$ is algebraically trivial. Every topological $\mathbb{R}$-vector bundle on $\mathbb{T}^{n}$, where $1 \leq n \leq 3$, is isomorphic to an algebraic $\mathbb{R}$-vector bundle, cf. [7]. Obviously, every topological $\mathbb{H}$-vector bundle on $\mathbb{T}^{n}$ is trivial, provided that $1 \leq n \leq 3$.

As usual, the $k$ th Chern class of a $\mathbb{C}$-vector bundle $\xi$ will be denoted by $c_{k}(\xi)$. Any $\mathbb{F}$-vector bundle $\eta$ can be regarded as a $\mathbb{K}$-vector bundle, denoted $\eta_{\mathbb{K}}$, where $\mathbb{K} \subseteq \mathbb{F}$ and $\mathbb{K}$ stands for $\mathbb{R}, \mathbb{C}$ or $\mathbb{H}$. In particular, $\eta_{\mathbb{F}}=\eta$. If $\eta$ is an $\mathbb{H}$-vector bundle, then $\eta_{\mathbb{R}}=\left(\eta_{\mathbb{C}}\right)_{\mathbb{R}}$.

Example 1.7 For any integer $n \geq 4$, there exists a pre-algebraic $\mathbb{H}$-line bundle $\eta$ on $\mathbb{T}^{n}$ such that the pre-algebraic $\mathbb{K}$-vector bundle $\eta_{\mathbb{K}}$ on $\mathbb{T}^{n}$ (where $\mathbb{K}$ stands for $\mathbb{R}, \mathbb{C}$ or $\mathbb{H}$ ) is not topologically isomorphic to any algebraic $\mathbb{K}$-vector bundle. This assertion can be proved as follows. As recalled in Example 1.6, each algebraic $\mathbb{C}$-vector bundle on $\mathbb{T}^{n}$ is stably trivial, and hence its $k$ th Chern class is equal to 0 for every $k \geq 1$. Let $\lambda$ be a topological $\mathbb{H}$-line bundle on $\mathbb{T}^{4}$ with $c_{2}\left(\lambda_{\mathbb{C}}\right) \neq 0$. According to Proposition 1.5 , one can assume that the bundle $\lambda$ is pre-algebraic. If $p: \mathbb{T}^{n}=\mathbb{T}^{4} \times \mathbb{T}^{n-4} \rightarrow \mathbb{T}^{4}$ is the canonical projection, then the 
$\mathbb{H}$-line bundle $\eta:=p^{*} \lambda$ on $\mathbb{T}^{n}$ is pre-algebraic and $c_{2}\left(\eta_{\mathbb{C}}\right) \neq 0$. Consequently, $\eta_{\mathbb{K}}$ is not topologically isomorphic to any algebraic $\mathbb{K}$-vector bundle, where $\mathbb{K}=\mathbb{C}$ or $\mathbb{K}=\mathbb{H}$. It remains to prove that $\eta_{\mathbb{R}}$ is not topologically isomorphic to any algebraic $\mathbb{R}$-vector bundle. Supposing otherwise, the complexification $\eta_{\mathbb{R}} \otimes \mathbb{C}$ of $\eta_{\mathbb{R}}$ would be topologically isomorphic to an algebraic $\mathbb{C}$-vector bundle, and hence $c_{2}\left(\eta_{\mathbb{R}} \otimes \mathbb{C}\right)=0$. However, one has $c !_{1}\left(\eta_{\mathbb{C}}\right)=0$, which implies $c_{2}\left(\eta_{\mathbb{R}} \otimes \mathbb{C}\right)=2 c_{2}\left(\eta_{\mathbb{C}}\right) \neq 0$, cf. [21, Corollary 15.5].

In real algebraic geometry, the role of regular, semi-algebraic and Nash maps is firmly established. Recently obtained results demonstrate that maps of a new type, called "continuous rational maps" in [19,20] and "applications régulues" in [12], are also very useful. It is now commonly accepted to call them regulous maps in English. Regulous maps are essential in the present paper (see Sect. 2 for the definition).

\section{Sections of pre-algebraic vector bundles}

Let $X$ be an affine real algebraic variety and let $\xi=(E, p, X)$ be a pre-algebraic $\mathbb{F}$-vector bundle on $X$. For a function $f: X \rightarrow \mathbb{R}$, let

$$
Z(f)=\{x \in X \mid f(x)=0\} .
$$

Assumption 2.1 Throughout this section the variety $X$ is assumed to be nonsingular.

Lemma 2.2 Let $f: X \rightarrow \mathbb{R}$ be a regular function. For any algebraic section $s: X \backslash Z(f) \rightarrow$ $E$, there exists a positive integer $d$ such that the map $u: X \rightarrow E$,

$$
u(x)=\left\{\begin{array}{ll}
f(x)^{d} s(x) & \text { for } x \in X \backslash Z(f) \\
0 & \text { for } x \in Z(f)
\end{array},\right.
$$

is a continuous section.

Proof Set $U=X \backslash Z(f)$. Let $\left\{U_{1}, \ldots, U_{q}\right\}$ be a Zariski open cover of $X$ such that the restriction $\mathbb{F}$-vector bundle $\xi_{\mid U_{i}}$ is algebraically trivial for $1 \leq i \leq q$. Let $\varphi_{i}: p^{-1}\left(U_{i}\right) \rightarrow$ $U_{i} \times \mathbb{F}^{r}$ be an algebraic trivialization of $\xi_{\mid U_{i}}$. The map

$$
\varphi_{i} \circ\left(s_{\mid U \cap U_{i}}\right): U \cap U_{i} \rightarrow U_{i} \times \mathbb{F}^{r}
$$

is regular. Since

$$
U \cap U_{i}=(X \backslash Z(f)) \cap U_{i}=U_{i} \backslash\left(Z(f) \cap U_{i}\right),
$$

there exists a positive integer $d$ such that the map $u_{i}: U_{i} \rightarrow E$,

$$
u_{i}(x)=\left\{\begin{array}{ll}
f(x)^{d} s(x) & \text { for } x \in U_{i} \backslash\left(Z(f) \cap U_{i}\right) \\
0 & \text { for } x \in Z(f) \cap U_{i}
\end{array},\right.
$$

is a continuous section for $1 \leq i \leq q$, cf. [5, Proposition 2.6.4]. By construction, $u_{\mid U_{i}}=u_{i}$, and hence $u$ is a continuous section.

Let $Y$ be a real algebraic variety (not necessarily affine). A map $f: X \rightarrow Y$ is said to be regulous if it is continuous and there exists a Zariski open and dense subset $U$ of $X$ such that the restriction $f_{\mid U}: U \rightarrow Y$ is a regular map. The term "regulous" corresponds to "régulue" used in [12]. Regulous maps are called continuous rational maps in [19,20]. In particular, the section $u$ in Lemma 2.2 is regulous. It should be mentioned that regulous maps with singular domains, which do not appear in the present paper, are defined in a different way, cf. [12]. 
Lemma 2.3 The $\mathbb{F}$-vector bundle $\xi$ is generated by global regulous sections, that is, there exist regulous sections $u_{1}: X \rightarrow E, \ldots, u_{n}: X \rightarrow E$ such that for each point $x$ in $X$, the vectors $u_{1}(x), \ldots, u_{n}(x)$ span the fiber of $\xi$ over $x$.

Proof It suffices to apply Lemma 2.2

Lemma 2.3 could also be deduced from Cartan's Theorem A for quasi-coherent regulous sheaves, cf. [12]. However, such a proof would be much more involved.

It is well known that multiblowups of $X$ can be used to transform regulous realvalued functions on $X$ into regular functions, cf. [12]. In the present section the following generalization of this result is needed.

Lemma 2.4 Let $Y$ be a real algebraic variety (not necessarily affine) and let $f: X \rightarrow Y$ be a regulous map. Assume that each point $x$ in $X$ has a Zariski open neighborhood $U_{x}$ in $X$ such that $f\left(U_{x}\right)$ is contained in a Zariski open affine subvariety $V_{x}$ of $Y$. Then there exists a multiblowup $\pi: X^{\prime} \rightarrow X$ of $X$ such that the composite map $f \circ \pi: X^{\prime} \rightarrow Y$ is regular.

Proof One can assume without loss of generality that the variety $X$ is irreducible. Let $U$ be a nonempty Zariski open subset of $X$ such that the restriction $f_{\mid U}: U \rightarrow Y$ is a regular map. Now, the proof will be divided into two steps.

Case 1 Assume that $Y=\mathbb{R}^{k}$ for some $k$. Consider $\mathbb{R}^{k}$ as a subset of real projective $k$-space $\mathbb{P}^{k}(\mathbb{R})$ and let $e: \mathbb{R}^{k} \hookrightarrow \mathbb{P}^{k}(\mathbb{R})$ be the inclusion map. The regular map $e \circ f_{\mid U}: U \rightarrow$ $\mathbb{P}^{k}(\mathbb{R})$ determines a rational map $\varphi: X \rightarrow \mathbb{P}^{k}(\mathbb{R})$. By Hironaka's theorem on resolution of indeterminacy points [14,18], there exist a multiblowup $\pi: X^{\prime} \rightarrow X$ and a regular map $g: X^{\prime} \rightarrow \mathbb{P}^{k}(\mathbb{R})$ satisfying $g=\varphi \circ \pi$ as rational maps. Consequently, since every nonempty Zariski open subset of $X^{\prime}$ is dense in the Euclidean topology, $g=e \circ f \circ \pi$ as continuous maps on $X^{\prime}$. This completes the proof for $Y=\mathbb{R}^{k}$.

Case 2 Suppose that $Y$ is an arbitrary real algebraic variety. Since $X$ is a quasi-compact topological space with the Zariski topology, one has $X=U_{x_{1}} \cup \cdots \cup U_{x_{q}}$ for some points $x_{1}, \ldots, x_{q}$ in $X$. Set $U_{i}=U_{x_{i}}$ and $V_{i}=V_{x_{i}}$, and denote by $f_{i}: U_{i} \rightarrow V_{i}$ the restriction of $f$ for $1 \leq i \leq q$. There exists a regular function $\lambda_{i}: X \rightarrow \mathbb{R}$ with $U_{i}=X \backslash Z\left(\lambda_{i}\right)$. Choose a regular embedding $\varepsilon_{i}: V_{i} \rightarrow \mathbb{R}^{n_{i}}$ for some positive integer $n_{i}$. The map $g_{i}:=\varepsilon_{i} \circ f_{i}$ : $U_{i} \rightarrow \mathbb{R}^{n_{i}}$ is continuous, and the restriction $g_{i \mid U \cap U_{i}}: U \cap U_{i} \rightarrow \mathbb{R}^{n_{i}}$ is a regular map. The set $U \cap U_{i}$ is Zariski dense in $U_{i}$, and hence it is also dense in the Euclidean topology, the variety $U_{i}$ being nonsingular. Consequently, the map $g_{i}$ is semi-algebraic, that is, its graph is semi-algebraic, cf. [5, Proposition 2.2.2]. It follows that there exists a positive integer $d$ such that the $\mathrm{m} !$ ap $h_{i}: X \rightarrow \mathbb{R}^{n_{i}}$,

$$
h_{i}(x)=\left\{\begin{array}{ll}
\lambda_{i}(x)^{d} g_{i}(x) & \text { for } x \in X \backslash Z\left(\lambda_{i}\right)=U_{i} \\
0 & \text { for } x \in Z\left(\lambda_{i}\right)
\end{array},\right.
$$

is continuous for $1 \leq i \leq q$. By construction, the map

$$
h=\left(h_{1}, \ldots, h_{q}\right): X \rightarrow \mathbb{R}^{n_{1}} \times \cdots \times \mathbb{R}^{n_{q}}
$$

is regulous. According to Case 1, there exists a multiblowup $\pi: X^{\prime} \rightarrow X$ of $X$ such that the composite map $h \circ \pi$ is regular.

Set $U_{i}^{\prime}=\pi^{-1}\left(U_{i}\right)$ and denote by $\pi_{i}: U_{i}^{\prime} \rightarrow U_{i}$ the restriction of $\pi$. Then $h_{i} \circ \pi_{i}=$ $\left(\lambda_{i} \circ \pi_{i}\right)^{d}\left(g_{i} \circ \pi_{i}\right)$, and hence the map $g_{i} \circ \pi_{i}: U_{i}^{\prime} \rightarrow \mathbb{R}^{n_{i}}$ is regular. Consequently, the map $f_{i} \circ \pi_{i}: U_{i}^{\prime} \rightarrow V_{i}$ is regular. Since the Zariski open sets $U_{1}^{\prime}, \ldots, U_{q}^{\prime}$ cover $X^{\prime}$, the map $f \circ \pi: X^{\prime} \rightarrow Y$ is regular. 
Lemma 2.5 Let $u_{1}: X \rightarrow E, \ldots, u_{n}: X \rightarrow E$ be regulous sections of the $\mathbb{F}$-vector bundle $\xi$. Then there exists a multiblowup $\pi: X^{\prime} \rightarrow X$ of $X$ such that the pullback sections $\pi^{*} u_{1}, \ldots, \pi^{*} u_{n}$ of the pullback $\mathbb{F}$-vector bundle $\pi^{*} \xi$ on $X^{\prime}$ are algebraic.

Proof Each point $x$ in $X$ has a Zariski open neighborhood $U_{x}$ such that the restriction $\mathbb{F}$-vector bundle $\xi_{\mid U_{x}}$ is algebraically trivial. Then $p^{-1}\left(U_{x}\right)$ is a Zariski open affine subvariety of $E$. By Lemma 2.4, there exists a multiblowup $\pi: X^{\prime} \rightarrow X$ such that the map $u_{i} \circ \pi: X^{\prime} \rightarrow E$ is regular for $1 \leq i \leq n$. Consequently, the pullback sections $\pi^{*} u_{1}, \ldots, \pi^{*} u_{n}$ of the pullback $\mathbb{F}$-vector bundle $\pi^{*} \xi$ are algebraic, as required.

Proof of Theorem 1.1 By Hironaka's resolution of singularities theorem [14,18], there exists a multiblowup $\sigma: Y \rightarrow X$ for which the variety $Y$ is nonsingular. For the proof one can replace $X$ and $\xi$ by $Y$ and $\sigma^{*} \xi$, respectively. Hence, from the beginning it can be assumed that the variety $X$ is nonsingular.

By Lemmas 2.3 and 2.5, there exists a multiblowup $\pi: X^{\prime} \rightarrow X$ of $X$ such that the pullback pre-algebraic $\mathbb{F}$-vector bundle $\pi^{*} \xi$ is generated by global algebraic sections. Consequently, $\pi^{*} \xi$ is an algebraic $\mathbb{F}$-vector bundle.

\section{Stiefel-Whitney classes of pre-algebraic vector bundles}

In this section it is proved that the Stiefel-Whitney classes do not distinguish pre-algebraic $\mathbb{R}$-vector bundles from algebraic $\mathbb{R}$-vector bundles.

Let $X$ be a compact nonsingular affine real algebraic variety. A homology class in $H_{d}(X ; \mathbb{Z} \backslash 2)$ is said to be algebraic if it can be represented by a $d$-dimensional algebraic subset of $X$, cf. [10] or [5,8]. The set $H_{d}^{\mathrm{alg}}(X ; \mathbb{Z} \backslash 2)$ of all algebraic homology classes in $H_{d}(X ; \mathbb{Z} \backslash 2)$ forms a subgroup. The subgroup $H_{\mathrm{alg}}^{k}(X ; \mathbb{Z} \backslash 2)$ of algebraic cohomology classes in $H^{k}(X ; \mathbb{Z} \backslash 2)$ is by definition the inverse image of $H_{n-k}^{\text {alg }}(X ; \mathbb{Z} \backslash 2)$ under the Poincaré duality isomorphism $H^{k}(X ; \mathbb{Z} \backslash 2) \rightarrow H_{n-k}(X ; \mathbb{Z} \backslash 2)$, where $n=\operatorname{dim} X$. The direct sum

$$
H_{\mathrm{alg}}^{*}(X ; \mathbb{Z} \backslash 2)=\bigoplus_{k \geq 0} H_{\mathrm{alg}}^{k}(X ; \mathbb{Z} \backslash 2)
$$

is a subring of the cohomology ring $H^{*}(X ; \mathbb{Z} \backslash 2)$, cf. [10] and [1,3]. The groups $H_{d}^{\text {alg }}(-; \mathbb{Z} \backslash 2)$ and $H_{\mathrm{alg}}^{k}(-; \mathbb{Z} \backslash 2)$ have the expected functorial property: If $f: X \rightarrow Y$ is a regular map between compact nonsingular affine real algebraic varieties, then

$$
f_{*}\left(H_{d}^{\mathrm{alg}}(X ; \mathbb{Z} \backslash 2)\right) \subseteq H_{d}^{\mathrm{alg}}(Y ; \mathbb{Z} \backslash 2) \text { and } f^{*}\left(H_{\mathrm{alg}}^{k}(Y ; \mathbb{Z} \backslash 2)\right) \subseteq H_{\mathrm{alg}}^{k}(X ; \mathbb{Z} \backslash 2)
$$

cf. [10] or [1,3]. Actually, a stronger result holds. The inclusions above are satisfied if $f$ is a regulous map, cf. [20, Proposition 1.3].

Proposition 3.1 Let $X, X^{\prime}$ be compact nonsingular affine real algebraic varieties and let $\varphi: X^{\prime} \rightarrow X$ be a birational regular map. If $u$ is a cohomology class in $H^{k}(X ; \mathbb{Z} \backslash 2)$ such that the cohomology class $\varphi^{*}(u)$ is algebraic, then $u$ also is algebraic.

Proof For any compact smooth manifold $M$ of dimension $n$, let $[M]$ denote its fundamental class in $H_{n}(M ; \mathbb{Z} \backslash 2)$. The Poincaré duality isomorphism $H^{k}(M ; \mathbb{Z} \backslash 2) \rightarrow H_{n-k}(M ; \mathbb{Z} \backslash 2)$ is given by $v \mapsto v \cap[M]$, where $\cap$ stands for the cap product. 
The topological degree modulo 2 of the map $\varphi: X^{\prime} \rightarrow X$ is 1 , and hence $\varphi_{*}\left(\left[X^{\prime}\right]\right)=[X]$. Consequently,

$$
\varphi_{*}\left(\varphi^{*}(u) \cap\left[X^{\prime}\right]\right)=u \cap \varphi_{*}\left(\left[X^{\prime}\right]\right)=u \cap[X] .
$$

By assumption, the homology class $\varphi^{*}(u) \cap\left[X^{\prime}\right]$ is algebraic. In view of the functoriality of $H_{d}^{\text {alg }}(-; \mathbb{Z} \backslash 2)$, the homology class $u \cap[X]$ is algebraic, and hence the cohomology class $u$ is algebraic.

It is well known that the Stiefel-Whitney classes of any algebraic $\mathbb{R}$-vector bundle are algebraic, cf. [10] or $[1,3]$. This result can be extended to pre-algebraic $\mathbb{R}$-vector bundles.

Theorem 3.2 Let $X$ be a compact nonsingular affine real algebraic variety. The StiefelWhitney classes of any pre-algebraic $\mathbb{R}$-vector bundle on $X$ are algebraic.

Proof Let $\xi$ be a pre-algebraic $\mathbb{R}$-vector bundle on $X$. According to Theorem 1.1, there exists a multiblowup $\pi: X^{\prime} \rightarrow X$ such that the $\mathbb{R}$-vector bundle $\pi^{*} \xi$ on $X^{\prime}$ is algebraic. Since the $k$ th Stiefel-Whitney class $w_{k}\left(\pi^{*} \xi\right)$ is algebraic and $w_{k}\left(\pi^{*} \xi\right)=\pi^{*}\left(w_{k}(\xi)\right)$, it follows from Proposition 3.1 that $w_{k}(\xi)$ also is algebraic for every $k \geq 0$.

Corollary 3.3 Let $X$ be a compact nonsingular affine real algebraic variety. If $\operatorname{dim} X \leq 3$, then any pre-algebraic $\mathbb{R}$-vector bundle on $X$ is topologically isomorphic to an algebraic $\mathbb{R}$-vector bundle.

Proof Assume that $\operatorname{dim} X \leq 3$. According to [7], a topological $\mathbb{R}$-vector bundle on $X$ is isomorphic to an algebraic $\mathbb{R}$-vector bundle if and only if it is of constant rank on each irreducible component of $X$ and if its Stiefel-Whitney classes are algebraic. Hence, it suffices to apply Theorem 3.2, the rank of any pre-algebraic vector bundle being locally constant for the Zariski topology.

It follows from Example 1.7 that Corollary 3.3 cannot be extended to varieties of dimension exceeding 3.

Theorem 3.2 is crucial in the proof of Proposition 1.4. It is convenient first to give the following:

Example 3.4 Let $n$ and $k$ be integers satisfying $0<k<n$. Let $\mathbb{T}^{n}=\mathbb{T}^{k} \times \mathbb{T}^{n-k}$ and let $y_{0}$ be a point in $\mathbb{T}^{n-k}$. Let $\alpha$ be the homology class in $H_{k}\left(\mathbb{T}^{n} ; \mathbb{Z} \backslash 2\right)$ represented by the smooth submanifold $K:=\mathbb{T}^{k} \times\left\{y_{0}\right\}$ of $\mathbb{T}^{n}$. Set

$$
A(n, k):=\left\{u \in H^{k}\left(\mathbb{T}^{n} ; \mathbb{Z} \backslash 2\right) \mid\langle u, \alpha\rangle=0\right\},
$$

where $\langle-,-\rangle$ stands for the Kronecker index (scalar product). Since the normal bundle of $K$ in $\mathbb{T}^{n}$ is trivial and $K$ is the boundary of a compact smooth manifold with boundary, it follows from [9, Proposition 2.5, Theorem 2.6] that there exist a nonsingular affine real algebraic variety $X$ and a smooth diffeomorphism $\varphi: X \rightarrow \mathbb{T}^{n}$ with

$$
H_{\mathrm{alg}}^{k}(X ; \mathbb{Z} \backslash 2) \subseteq \varphi^{*}(A(n, k)) .
$$

Proof of Proposition 1.4 Let $\mathbb{P}^{1}(\mathbb{F})$ be the projective $\mathbb{F}$-line and let $\gamma^{1}(\mathbb{F})$ be the tautological $\mathbb{F}$-line bundle on $\mathbb{P}^{1}(\mathbb{F})$. Recall that $w_{d(\mathbb{F})}\left(\gamma^{1}(\mathbb{F})_{\mathbb{R}}\right) \neq 0$ in $H^{d(\mathbb{F})}\left(\mathbb{P}^{1}(\mathbb{F}) ; \mathbb{Z} \backslash 2\right)$. Choosing a continuous map $\psi: \mathbb{T}^{d(\mathbb{F})} \rightarrow \mathbb{P}^{1}(\mathbb{F})$ for which the induced homomorphism

$$
\psi^{*}: H^{d(\mathbb{F})}\left(\mathbb{P}^{1}(\mathbb{F}) ; \mathbb{Z} \backslash 2\right) \rightarrow H^{d(\mathbb{F})}\left(\mathbb{T}^{d(\mathbb{F})} ; \mathbb{Z} \backslash 2\right)
$$


is an isomorphism, one obtains an $\mathbb{F}$-line bundle $\lambda:=\psi^{*} \gamma^{1}(\mathbb{F})$ on $\mathbb{T}^{d(\mathbb{F})}$ with $w_{d(\mathbb{F})}\left(\lambda_{\mathbb{R}}\right) \neq 0$. If $n>d(\mathbb{F})$ and $p: \mathbb{T}^{n}=\mathbb{T}^{d(\mathbb{F})} \times \mathbb{T}^{n-d(\mathbb{F})} \rightarrow \mathbb{T}^{d(\mathbb{F})}$ is the canonical projection, then for the $\mathbb{F}$-line bundle $\eta:=p^{*} \lambda$ on $\mathbb{T}^{n}$ one gets

$$
w_{d(\mathbb{F})}\left(\eta_{\mathbb{R}}\right) \notin A(n, d(\mathbb{F})),
$$

where $A(n, d(\mathbb{F}))$ is the subgroup of $H^{d(\mathbb{F})}\left(\mathbb{T}^{n} ; \mathbb{Z} \backslash 2\right)$ defined in Example 3.4. Choose a nonsingular affine real algebraic variety $X$ and a smooth diffeomorphism $\varphi: X \rightarrow \mathbb{T}^{n}$ such that

$$
H_{\mathrm{alg}}^{d(\mathbb{F})}(X ; \mathbb{Z} \backslash 2) \subseteq \varphi^{*}(A(n, d(\mathbb{F}))) .
$$

By construction, for the $\mathbb{F}$-line bundle $\xi:=\varphi^{*} \eta$ on $X$ one has

$$
w_{d(\mathbb{F})}\left(\xi_{\mathbb{R}}\right) \notin \varphi^{*}(A(n, d(\mathbb{F}))),
$$

and hence

$$
w_{d(\mathbb{F})}\left(\xi_{\mathbb{R}}\right) \notin H_{\mathrm{alg}}^{d(\mathbb{F})}(X ; \mathbb{Z} \backslash 2) .
$$

In other words, the Stiefel-Whitney class $w_{d(\mathbb{F})}\left(\xi_{\mathbb{R}}\right)$ is not algebraic. According to Theorem 3.2, the topological $\mathbb{F}$-line bundle $\xi$ on $X$ cannot be isomorphic to any pre-algebraic $\mathbb{F}$-line bundle.

\section{A construction of pre-algebraic vector bundles}

A certain construction will prove to be very useful for the purpose of comparison of prealgebraic, algebraic and topological $\mathbb{F}$-vector bundles. First some topological facts will be recalled for the convenience of the reader.

Let $M$ be a smooth (of class $\mathcal{C}^{\infty}$ ) manifold and let $N$ be a smooth submanifold of codimension $k$. By convention, submanifolds are assumed to be closed subsets of the ambient manifold. Assume that the normal bundle of $N$ in $M$ is oriented and denote by $\tau_{N}^{M}$ the Thom class of $N$ in the cohomology group $H^{k}(M, M \backslash N ; \mathbb{Z})$, cf. [21, p. 118]. The image of $\tau_{N}^{M}$ by the restriction homomorphism $H^{k}(M, M \backslash N ; \mathbb{Z}) \rightarrow H^{k}(M ; \mathbb{Z})$, induced by the inclusion map $M \hookrightarrow(M, M \backslash N)$, will be denoted by $[N]^{M}$ and called the cohomology class represented by $N$. If $M$ is compact and oriented, and $N$ is endowed with the compatible orientation, then $[N]^{M}$ is up to sign Poincaré dual to the homology class in $H_{*}(M ; \mathbb{Z})$ represented by $N$, cf. [21, p.136].

Let $P$ be a smooth manifold and let $Q$ be a smooth submanifold of $P$. Let $f: M \rightarrow P$ be a smooth map transverse to $Q$. If the normal bundle of $Q$ in $P$ is oriented and the normal bundle of the smooth submanifold $N:=f^{-1}(Q)$ of $M$ is endowed with the orientation induced by $f$, then $\tau_{N}^{M}=f^{*}\left(\tau_{Q}^{P}\right)$, where $f$ is regarded as a map from $(M, M \backslash N)$ into $(P, P \backslash Q)$ (this follows from [15, p. 117, Theorem 6.7]). In particular, $[N]^{M}=f^{*}\left([Q]^{P}\right)$.

Let $\xi=(E, p, M)$ be an oriented smooth $\mathbb{R}$-vector bundle on $M$ of rank $k$. For any smooth section $s: M \rightarrow E$, let $Z(s)$ denote the zero locus of $s$,

$$
Z(s)=\{x \in M \mid s(x)=0\} .
$$

If the section $s$ is transverse to the zero section and the normal bundle of $Z(s)$ in $M$ is endowed with the orientation induced by $s$ from the orientation of $\xi$, then

$$
e(\xi)=[Z(s)]^{M},
$$


where $e(\xi)$ stands for the Euler class of $\xi$. Indeed, identify $M$ with the image of the zero section of $\xi$. The section $s$ is transverse to $M$ and $Z(s)=s^{-1}(M)$. Consequently, $[Z(s)]^{M}=$ $s^{*}\left([M]^{E}\right)$. Hence,

$$
p^{*}\left([Z(s)]^{M}=p^{*}\left(s^{*}\left([M]^{E}\right)\right)=(s \circ p)^{*}\left([M]^{E}\right)=[M]^{E},\right.
$$

where the last equality holds since $s \circ p: E \rightarrow E$ is homotopic to the identity map. On the other hand, $p^{*}(e(\xi))=[M]^{E}$, cf. [21, p. 98]. It follows that $e(\xi)=[Z(s)]^{M}$ since $p^{*}$ is an isomorphism.

Let $\mathbb{K}$ stand for $\mathbb{C}$ or $\mathbb{H}$, and let $d(\mathbb{K})=\operatorname{dim}_{\mathbb{R}} \mathbb{K}$. By [21, p. 158], for any $\mathbb{K}$-vector bundle $\xi$ of rank $r$,

$$
e\left(\xi_{\mathbb{R}}\right)=c_{k}\left(\xi_{\mathbb{C}}\right),
$$

where $k=\frac{1}{2} r d(\mathbb{K})$. The notation $\xi_{\mathbb{R}}$ and $\xi_{\mathbb{C}}$ is introduced in Sect. 1 .

Proposition 4.1 Let $X$ be a compact nonsingular affine real algebraic variety and let $Y$ be a nonsingular Zariski closed subvariety of $X$ of codimension $d(\mathbb{K})$. Assume that the normal bundle of $Y$ in $X$ is trivial (as a topological $\mathbb{R}$-vector bundle) and endowed with an orientation. Then there exists a pre-algebraic $\mathbb{K}$-line bundle $\xi$ on $X$ with $c_{k}\left(\xi_{\mathbb{C}}\right)=[Y]^{X}$, where $k=\frac{1}{2} d(\mathbb{K})$.

Proof Let $T$ be a tubular neighborhood of $Y$ in $X$. Since the normal bundle of $Y$ in $X$ is trivial, there exists a smooth function $h: T \rightarrow \mathbb{K}$ such that $h$ is transverse to 0 in $\mathbb{K}, h^{-1}(0)=Y$ and the given orientation of the normal bundle of $Y$ in $X$ coincides with the orientation induced by $h$ from the canonical orientation of $\mathbb{K}=\mathbb{R}^{d(\mathbb{K})}$. One can find a smooth function $g: X \rightarrow \mathbb{K}$ such that $g=h$ in a neighborhood of $Y$ and $g$ is transverse to 0 in $\mathbb{K}$. By a relative version of the Weierstrass approximation theorem [5, Lemma 12.5.5], there exists a regular function $f: X \rightarrow \mathbb{K}$ that is arbitrarily close to $g$ in the $\mathcal{C}^{\infty}$ topology and $Y \subseteq f^{-1}(0)$. If $f$ is sufficiently close to $g$, then $f$ is transverse to 0 in $\mathbb{K}$ and $f^{-1}(0)=Y \cup Y^{\prime}$ with $Y \cap Y^{\prime}=\emptyset$. By [5, Proposition 3.3.17], $Y^{\prime}$ is a Zariski closed subset of $X$. The sets $U_{1}=X \backslash Y$ and $U_{2}=X \backslash Y^{\prime}$ form a Zariski open cover of $X$. Let $\xi=(E, p, X)$ be the pre-algebraic $\mathbb{K}$-line bundle on $X$, where $E$ is obtained by gluing $U_{1} \times \mathbb{K}$ and $U_{2} \times \mathbb{K}$ over $U_{1} \cap U_{2}$ via the regular map $f_{\mid U_{1} \cap U_{2}}: U_{1} \cap U_{2} \rightarrow \mathbb{K} \backslash\{0\}\left(\left(1, x_{1}, v_{1}\right) \in\{1\} \times U_{1} \times \mathbb{K}\right.$ is identified with $\left(2, x_{2}, v_{2}\right) \in\{2\} \times U_{2} \times \mathbb{K}$ if and only if $x_{1}=x_{2}$ is in $U_{1} \cap U_{2}$ and $\left.f\left(x_{1}\right) v_{1}=v_{2}\right)$, and $p: E \rightarrow X$ is the obvious projection, cf. [5, p.299]. The sections

$$
\begin{aligned}
& s_{1}: U_{1} \rightarrow U_{1} \times \mathbb{K}, \quad s_{1}(x)=(x, 1) \\
& s_{2}: U_{2} \rightarrow U_{2} \times \mathbb{K}, \quad s_{2}(x)=(x, f(x))
\end{aligned}
$$

determine an algebraic section $s: X \rightarrow E$ such that $s$ is transverse to the zero section, $Z(s)=$ $Y$ and the given orientation of the normal bundle of $Y$ in $X$ coincides with the orientation induced by $s$ from the canonical orientation of $\xi_{\mathbb{R}}$. Consequently, $c_{k}\left(\xi_{\mathbb{C}}\right)=e\left(\xi_{\mathbb{R}}\right)=[Y]^{X}$, where $k=\frac{1}{2} d(\mathbb{K})$.

Proof of Proposition 1.5 Any cohomology class in $H^{d(\mathbb{F})}(X ; \mathbb{Z})$ is of the form $[Y]^{X}$, where $Y$ is a finite subset of $X$ and the normal bundle of $Y$ in $X$ is suitably oriented. It remains to consider $\mathbb{F}=\mathbb{C}$ and $\mathbb{F}=\mathbb{H}$. In these cases, by Proposition 4.1, there exists a pre-algebraic $\mathbb{F}$-vector bundle $\xi$ on $X$ with $c_{k}\left(\xi_{\mathbb{C}}\right)=[Y]^{X}$, where $k=\frac{1}{2} d(\mathbb{F})$.

If $\eta$ is a topological $\mathbb{F}$-line bundle on $X$ with $c_{k}\left(\eta_{\mathbb{C}}\right)=[Y]^{X}$, then $\eta$ is isomorphic to $\xi$. Indeed, this is obvious for $\mathbb{F}=\mathbb{C}$ since topological $\mathbb{C}$-line bundles are classified by the first Chern class. If $\mathbb{F}=\mathbb{H}$, then $\operatorname{dim} X=4$ and hence $\eta$ is isomorphic to an $\mathbb{H}$-line bundle of 
the form $f^{*} \gamma^{1}(\mathbb{H})$, where $\gamma^{1}(\mathbb{H})$ is the tautological $\mathbb{H}$-line bundle on the projective $\mathbb{H}$-line $\mathbb{P}^{1}(\mathbb{H})$ and $f: X \rightarrow \mathbb{P}^{1}(\mathbb{H})$ is a continuous map. Since $c_{2}\left(\gamma^{1}(\mathbb{H})_{\mathbb{C}}\right)$ is a generator of the cohomology group $H^{4}\left(\mathbb{P}^{1}(\mathbb{H}) ; \mathbb{Z}\right) \cong \mathbb{Z}$, by Hopf's theorem, the homotopy class of $f$ is determined by the cohomology class $f^{*}\left(c_{2}\left(\gamma^{1}(\mathbb{H})_{\mathbb{C}}\right)\right)=c_{2}\left(\eta_{\mathbb{C}}\right)$. Thus, topological $\mathbb{H}-$-line bundles on $X$ are classified by the second Chern class. The proof is complete.

Example 4.2 As recalled in Example 1.6, any algebraic $\mathbb{C}$-line bundle on $\mathbb{T}^{n}$ is algebraically trivial. However, any topological $\mathbb{C}$-line bundle on $\mathbb{T}^{n}$ is isomorphic to a pre-algebraic $\mathbb{C}$-line bundle. The last assertion can be proved as follows. It suffices to consider $n \geq 2$. The cohomology group $H^{2}\left(\mathbb{T}^{n} ; \mathbb{Z}\right)$ is generated by elements of the form $[Y]^{\mathbb{T}^{n}}$, where $Y$ is a nonsingular codimension 2 Zariski closed subvariety of $\mathbb{T}^{n}$ with trivial and oriented normal bundle. By Proposition 4.1, there exists a pre-algebraic $\mathbb{C}$-line bundle $\xi$ on $\mathbb{T}^{n}$ with $c_{1}(\xi)=[Y]^{\mathbb{T}^{n}}$. Note that the dual bundle $\xi^{\vee}$ is pre-algebraic and satisfies $c_{1}\left(\xi^{\vee}\right)=-[Y]^{\mathbb{T}^{n}}$. Consequently, any element of $H^{2}\left(\mathbb{T}^{n} ; \mathbb{Z}\right)$ can be written as

$$
n_{1} c_{1}\left(\xi_{1}\right)+\cdots+n_{r} c_{1}\left(\xi_{r}\right)=c_{1}\left(\xi_{1}^{\otimes n_{1}} \otimes \cdots \otimes \xi_{r}^{\otimes n_{r}}\right)
$$

for some pre-algebraic $\mathbb{C}$-line bundles $\xi_{i}$ on $\mathbb{T}^{n}$ and some nonnegative integers $n_{i}$ with $1 \leq i \leq r$. The assertion follows since topological $\mathbb{C}$-line bundles are classified by the first Chern class.

\section{Chern classes and Pontryagin classes of pre-algebraic vector bundles}

In this section the theory of Chern and Pontryagin classes for pre-algebraic vector bundles is developed and contrasted with the already known results for algebraic vector bundles.

Let $X$ be a compact nonsingular affine real algebraic variety. A nonsingular projective complexification of $X$ is a pair $(V, \varepsilon)$, where $V$ is a nonsingular projective scheme over $\mathbb{R}$ and $\varepsilon: X \rightarrow V(\mathbb{C})$ is an injective map such that $V(\mathbb{R})$ is Zariski dense in $V, \varepsilon(X)=V(\mathbb{R})$ and $\varepsilon$ is a biregular isomorphism from $X$ onto $V(\mathbb{R})$. Here the set $V(\mathbb{R})$ of real points of $V$ is regarded as a subset of the set $V(\mathbb{C})$ of complex points of $V$. The existence of $(V, \varepsilon)$ follows from Hironaka's resolution of singularities theorem $[14,18]$. The set of complex points of the scheme $V_{\mathbb{C}}=V \times_{\mathbb{R}} \mathbb{C}$ over $\mathbb{C}$ is identified with $V(\mathbb{C})$. The cycle map

$$
c l_{\mathbb{C}}: A^{*}\left(V_{\mathbb{C}}\right)=\bigoplus_{k \geq 0} A^{k}\left(V_{\mathbb{C}}\right) \rightarrow H^{\mathrm{even}}(V(\mathbb{C}) ; \mathbb{Z})=\bigoplus_{k \geq 0} H^{2 k}(V(\mathbb{C}) ; \mathbb{Z})
$$

is a ring homomorphism defined on the Chow ring of $V_{\mathbb{C}}$, cf. [10] or [13, Corollary 19.2] Hence,

$$
H_{\mathrm{alg}}^{2 k}(V(\mathbb{C}) ; \mathbb{Z}):=\operatorname{cl}_{\mathbb{C}}\left(A^{k}\left(V_{\mathbb{C}}\right)\right)
$$

is the subgroup of $H^{2 k}(V(\mathbb{C}) ; \mathbb{Z})$ that consists of the cohomology classes corresponding to algebraic cycles (defined over $\mathbb{C}$ ) on $V_{\mathbb{C}}$ of codimension $k$. By construction,

$$
H_{\mathbb{C}-\text { alg }}^{2 k}(X ; \mathbb{Z}):=\varepsilon^{*}\left(H_{\text {alg }}^{2 k}(V(\mathbb{C}) ; \mathbb{Z})\right)
$$

is a subgroup of $H^{2 k}(X ; \mathbb{Z})$, and

$$
H_{\mathbb{C} \text {-alg }}^{\text {even }}(X ; \mathbb{Z}):=\bigoplus_{k \geq 0} H_{\mathbb{C} \text {-alg }}^{2 k}(X ; \mathbb{Z})
$$


is a subring of $H^{\text {even }}(X ; \mathbb{Z})$. The subring $H_{\mathbb{C} \text {-alg }}^{\text {even }}(X ; \mathbb{Z})$ does not depend on the choice of $(V, \varepsilon)$, cf. [4, p. 278]. A cohomology class in $H^{2 k}(X ; \mathbb{Z})$ is said to be $\mathbb{C}$-algebraic if it belongs to $H_{\mathbb{C}-\text { alg }}^{2 k}(X ; \mathbb{Z})$.

Example 5.1 For any positive integer $n$,

$$
H_{\mathbb{C}-\text { alg }}^{\text {even }}\left(\mathbb{T}^{n} ; \mathbb{Z}\right)=H^{0}\left(\mathbb{T}^{n} ; \mathbb{Z}\right) .
$$

Indeed, $\mathbb{S}^{1}$ can be identified with the real projective line $\mathbb{P}^{1}(\mathbb{R})$. If $\mathbb{P}^{1}=\operatorname{Proj}\left(\mathbb{R}\left[X_{0}, X_{1}\right]\right)$ and $i: \mathbb{P}^{1}(\mathbb{R}) \hookrightarrow \mathbb{P}^{1}(\mathbb{C})$ is the inclusion map, then the pair $\left(\mathbb{P}^{1} \times \mathbb{R} \cdots \times \mathbb{R}^{1}, i \times \cdots \times i\right)$ of the $n$-fold products is a nonsingular projective complexification of $\mathbb{T}^{n}$. The assertion follows since $\mathbb{P}^{1}(\mathbb{C})$ is homeomorphic to the unit 2-sphere.

It is well known that the Chern classes of any algebraic $\mathbb{C}$-vector bundle and the Pontryagin classes of any algebraic $\mathbb{R}$-vector bundle are $\mathbb{C}$-algebraic, cf. [4, Theorem 5.3]. The following example shows that this result cannot be extended to pre-algebraic vector bundles.

Example 5.2 Let $\eta$ be the pre-algebraic $\mathbb{H}$-line bundle on $\mathbb{T}^{n}$ constructed in Example 1.7. Recall that $c_{2}\left(\eta_{\mathbb{C}}\right) \neq 0$ and $c_{2}\left(\eta_{\mathbb{R}} \otimes \mathbb{C}\right) \neq 0$. Since the first Pontryagin class $p_{1}\left(\eta_{\mathbb{R}}\right)$ is defined to be $-c_{2}\left(\eta_{\mathbb{R}} \otimes \mathbb{C}\right)$, one gets $p_{1}\left(\eta_{\mathbb{R}}\right) \neq 0$. According to Example 5.1, the Chern class $c_{2}\left(\eta_{\mathbb{C}}\right)$ and the Pontryagin class $p_{1}\left(\eta_{\mathbb{R}}\right)$ are not $\mathbb{C}$-algebraic.

There is no counterpart of Proposition 3.1 for $\mathbb{C}$-algebraic cohomology classes. To see this, it suffices to combine Example 5.1 with the following:

Example 5.3 There exists a multiblowup $\pi: \tilde{\mathbb{T}}^{n} \rightarrow \mathbb{T}^{n}$ of $\mathbb{T}^{n}$ satisfying

$$
\pi^{*}\left(H^{2}\left(\mathbb{T}^{n} ; \mathbb{Z}\right)\right) \subseteq H_{\mathbb{C}-\text { alg }}^{2}\left(\tilde{\mathbb{T}}^{n} ; \mathbb{Z}\right) .
$$

Indeed, let $v_{1}, \ldots, v_{q}$ be cohomology classes that generate the group $H^{2}\left(\mathbb{T}^{n} ; \mathbb{Z}\right)$. Each $v_{i}$ can be expressed as $v_{i}=c_{1}\left(\xi_{i}\right)$ for some topological $\mathbb{C}$-line bundle $\xi_{i}$ on $\mathbb{T}^{n}$. In view of Example 4.2, the bundle $\xi_{i}$ can be assumed to be pre-algebraic. By Theorem 1.1, one can find a multiblowup $\pi: \tilde{\mathbb{T}}^{n} \rightarrow \mathbb{T}^{n}$ of $\mathbb{T}^{n}$ such that the pullback $\mathbb{C}$-vector bundle $\pi^{*} \xi_{i}$ on $\tilde{\mathbb{T}}^{n}$ is algebraic for $1 \leq i \leq q$ (induction on $q$ ). Consequently, the cohomology class

$$
c_{1}\left(\pi^{*} \xi_{i}\right)=\pi^{*}\left(c_{1}(\xi)\right)=\pi^{*}\left(v_{i}\right)
$$

is $\mathbb{C}$-algebraic. Hence, the inclusion holds.

The groups $H_{\mathbb{C} \text {-alg }}^{2 k}(-; \mathbb{Z})$ have the following functorial property: if $f: X \rightarrow Y$ is a regular map between compact nonsingular affine real algebraic varieties, then

$$
f^{*}\left(H_{\mathbb{C}-\text { alg }}^{2 k}(Y ; \mathbb{Z})\right) \subseteq H_{\mathbb{C}-\text { alg }}^{2 k}(X ; \mathbb{Z})
$$

for every $k \geq 0$, cf. [4, Proposition 3.1]. The next example shows that the inclusion above may not hold if $f$ is a regulous map.

Let $\mathbb{S}^{n}$ be the unit $n$-sphere,

$$
\mathbb{S}^{n}=\left\{\left(x_{1}, \ldots, x_{n+1}\right) \in \mathbb{R}^{n+1} \mid x_{1}^{2}+\cdots+x_{n+1}^{2}=1\right\} .
$$

According to [4, Proposition 4.8],

$$
H_{\mathbb{C}-\text { alg }}^{2 k}\left(\mathbb{S}^{2 k} ; \mathbb{Z}\right)=H^{2 k}\left(\mathbb{S}^{2 k} ; \mathbb{Z}\right)
$$

for every $k \geq 1$. 
Example 5.4 By [20, Theorem 2.4], each continuous map from $\mathbb{T}^{n}$ into $\mathbb{S}^{n}$ is homotopic to a regulous map. Hence, there exists a regulous map $f: \mathbb{T}^{n} \rightarrow \mathbb{S}^{n}$ such that the induced homomorphism

$$
f^{*}: H^{n}\left(\mathbb{S}^{n} ; \mathbb{Z}\right) \rightarrow H^{n}\left(\mathbb{T}^{n} ; \mathbb{Z}\right)
$$

is an isomorphism. If $n=2 k$ is a positive even integer, then $f^{*}\left(H_{\mathbb{C}-\text { alg }}^{2 k}\left(\mathbb{S}^{2 k} ; \mathbb{Z}\right)\right)$ is not contained in $H_{\mathbb{C} \text {-alg }}^{2 k}\left(\mathbb{T}^{2 k} ; \mathbb{Z}\right)$. Indeed, $H_{\mathbb{C} \text {-alg }}^{2 k}\left(\mathbb{S}^{2 k} ; \mathbb{Z}\right)=H^{2 k}\left(\mathbb{S}^{2 k} ; \mathbb{Z}\right) \cong \mathbb{Z}$, whereas $H_{\mathbb{C}-\text { alg }}^{2 k}\left(\mathbb{T}^{2 k} ; \mathbb{Z}\right)=0$ in view of Example 5.1.

Examples 5.2, 5.3 and 5.4 suggest the following:

Definition 5.5 A cohomology class $u$ in $H^{2 k}(X ; \mathbb{Z})$, where $X$ is a compact nonsingular affine real algebraic variety, is said to be blow- $\mathbb{C}$-algebraic if there exists a multiblowup $\pi: X^{\prime} \rightarrow X$ such that the cohomology class $\pi^{*}(u)$ is $\mathbb{C}$-algebraic.

The next task is to prove that the set

$$
\bar{H}_{\mathbb{C}-\text { alg }}^{2 k}(X ; \mathbb{Z})
$$

of all blow- $\mathbb{C}$-algebraic cohomology classes in $H^{2 k}(X ; \mathbb{Z})$ has suitable properties.

Proposition 5.6 The set $\bar{H}_{\mathbb{C}-a l g}^{2 k}(X ; \mathbb{Z})$ forms a subgroup of $H^{2 k}(X ; \mathbb{Z})$. Furthermore, the direct sum

$$
\bar{H}_{\mathbb{C} \text {-alg }}^{\text {even }}(X ; \mathbb{Z}):=\bigoplus_{k \geq 0} \bar{H}_{\mathbb{C} \text {-alg }}^{2 k}(X ; \mathbb{Z})
$$

is a subring of $H^{\mathrm{even}}(X ; \mathbb{Z})$.

Proof Obviously, it suffices to prove that the set $\bar{H}_{\mathbb{C} \text {-alg }}^{2 k}(X ; \mathbb{Z})$ is closed under addition, and the set $\bar{H}_{\mathbb{C} \text {-alg }}^{\text {even }}(X ; \mathbb{Z})$ is closed under multiplication.

Let $u_{1}$ and $u_{2}$ be cohomology classes in $\bar{H}_{\mathbb{C} \text {-alg }}^{2 k}(X ; \mathbb{Z})$. By definition, there exists a multiblowup $\pi_{i}: X_{i} \rightarrow X$ such that the cohomology class $\pi_{i}^{*}\left(u_{i}\right)$ is in $H_{\mathbb{C}-a l g}^{2 k}\left(X_{i} ; \mathbb{Z}\right)$ for $i=1,2$. Consider the rational map $\pi_{1}^{-1} \circ \pi_{2}: X_{2} \rightarrow X_{1}$. By Hironaka's theorem on resolution of indeterminacy points [14,18], there exist a multiblowup $\pi_{3}: X_{3} \rightarrow X_{2}$ and a regular map $\varphi: X_{3} \rightarrow X_{1}$ such that $\pi_{1}^{-1} \circ \pi_{2} \circ \pi_{3}=\varphi$ as rational maps. Consequently $\pi_{2} \circ \pi_{3}=\pi_{1} \circ \varphi$, and hence

$$
\begin{aligned}
\left(\pi_{2} \circ \pi_{3}\right)^{*}\left(u_{1}+u_{2}\right) & =\left(\pi_{1} \circ \varphi\right)^{*}\left(u_{1}\right)+\left(\pi_{2} \circ \pi_{3}\right)^{*}\left(u_{2}\right) \\
& =\varphi^{*}\left(\pi_{1}^{*}\left(u_{1}\right)\right)+\pi_{3}^{*}\left(\pi_{2}^{*}\left(u_{2}\right)\right) .
\end{aligned}
$$

The functoriality of $H_{\mathbb{C}-a l g}^{2 k}(-; \mathbb{Z})$ for regular maps implies that the cohomology class $\left(\pi_{2} \circ \pi_{3}\right)^{*}\left(u_{1}+u_{2}\right)$ is in $H_{\mathbb{C}-a l g}^{2 k}\left(X_{3} ; \mathbb{Z}\right)$. Thus, $u_{1}+u_{2}$ is in $\bar{H}_{\mathbb{C}-a l g}^{2 k}(X ; \mathbb{Z})$.

A straightforward modification of the argument just given shows that the set $\bar{H}_{\mathbb{C} \text {-alg }}^{\text {even }}(X ; \mathbb{Z})$ is closed under multiplication.

Theorem 5.7 Let $X$ be a compact nonsingular affine real algebraic variety. The Chern classes of any pre-algebraic $\mathbb{C}$-vector bundle on $X$ and the Pontryagin classes of any prealgebraic $\mathbb{R}$-vector bundle on $X$ are blow- $\mathbb{C}$-algebraic. 
Proof Let $\xi$ be a pre-algebraic $\mathbb{C}$-vector bundle on $X$. According to Theorem 1.1 , there exists a multiblowup $\pi: X^{\prime} \rightarrow X$ such that the $\mathbb{C}$-vector bundle $\pi^{*} \xi$ on $X^{\prime}$ is $\mathbb{C}$-algebraic. Since the Chern class $c_{k}\left(\pi^{*} \xi\right)$ is $\mathbb{C}$-algebraic and $c_{k}\left(\pi^{*} \xi\right)=\pi^{*}\left(c_{k}(\xi)\right)$, the class $c_{k}(\xi)$ is blow- $\mathbb{C}$-algebraic for every $k \geq 0$.

If $\eta$ is a pre-algebraic $\mathbb{R}$-vector bundle on $X$, then its $k$ th Pontryagin class $p_{k}(\eta)$ is blow$\mathbb{C}$-algebraic since $p_{k}(\eta)=(-1)^{k} c_{2 k}(\eta \otimes \mathbb{C})$.

The groups $\bar{H}_{\mathbb{C} \text {-alg }}^{2 k}(-; \mathbb{Z})$ are functorial for surjective regulous maps. More precisely:

Theorem 5.8 If $f: X \rightarrow Y$ is a surjective regulous map between compact affine nonsingular real algebraic varieties, then

$$
f^{*}\left(\bar{H}_{\mathbb{C}-\text { alg }}^{2 k}(Y ; \mathbb{Z})\right) \subseteq \bar{H}_{\mathbb{C} \text {-alg }}^{2 k}(X ; \mathbb{Z})
$$

for every $k \geq 0$.

Proof Let $v$ be a cohomology class in $\bar{H}_{\mathbb{C} \text {-alg }}^{2 k}(Y ; \mathbb{Z})$. Let $\sigma: Y^{\prime} \rightarrow Y$ be a multiblowup such that $\sigma^{*}(v)$ is in $H_{\mathbb{C} \text {-alg }}^{2 k}\left(Y^{\prime} ; \mathbb{Z}\right)$. Consider the rational map $\sigma^{-1} \circ f: X \rightarrow Y^{\prime}$. By Hironaka's theorem on resolution of indeterminacy points [14,18], there exist a multiblowup $\pi: X^{\prime} \rightarrow X$ and a regular map $f^{\prime}: X^{\prime} \rightarrow Y^{\prime}$ such that $\sigma^{-1} \circ f \circ \pi=f^{\prime}$ as rational maps. Consequently, $f \circ \pi=\sigma \circ f^{\prime}$, and hence

$$
\pi^{*}\left(f^{*}(v)\right)=(f \circ \pi)^{*}(v)=\left(\sigma \circ f^{\prime}\right)^{*}(v)=\left(f^{\prime}\right)^{*}\left(\sigma^{*}(v)\right) .
$$

In view of the functoriality of the groups $H_{\mathbb{C}-a l g}^{2 k}(-; \mathbb{Z})$ for regular maps, the cohomology class $\left(f^{\prime}\right)^{*}\left(\sigma^{*}(v)\right)$ is in $H_{\mathbb{C} \text {-alg }}^{2 k}\left(X^{\prime} ; \mathbb{Z}\right)$. Thus, $f^{*}(v)$ is in $\bar{H}_{\mathbb{C} \text {-alg }}^{2 k}(X ; \mathbb{Z})$, as required.

There is a counterpart of Proposition 3.1 for blow- $\mathbb{C}$-algebraic cohomology classes.

Proposition 5.9 Let $X$ and $X^{\prime}$ be compact nonsingular affine real algebraic varieties and let $\varphi: X^{\prime} \rightarrow X$ be a birational regular map. If $u$ is a cohomology class in $H^{2 k}(X ; \mathbb{Z})$ such that the cohomology class $\varphi^{*}(u)$ is blow- $\mathbb{C}$-algebraic, then $u$ also is blow- $\mathbb{C}$-algebraic.

Proof Let $\sigma: Y \rightarrow X^{\prime}$ be a multiblowup such that the cohomology class $\sigma^{*}\left(\varphi^{*}(u)\right)=$ $(\varphi \circ \sigma)^{*}(u)$ is $\mathbb{C}$-algebraic. Set $\psi=\varphi \circ \sigma$ and consider the rational map $\psi^{-1}: X \rightarrow Y$. By Hironaka's theorem on resolution of indeterminacy points $[14,18]$, there exist a multiblowup $\pi: X^{\prime \prime} \rightarrow X$ and a regular map $f: X^{\prime \prime} \rightarrow Y$ such that $\psi^{-1} \circ \pi=f$ as rational maps. Consequently, $\pi=\psi \circ f=\varphi \circ \sigma \circ f$, and hence

$$
\pi^{*}(u)=(\varphi \circ \sigma \circ f)^{*}(u)=f^{*}\left((\varphi \circ \sigma)^{*}(u)\right) .
$$

The cohomology class $f^{*}\left((\varphi \circ \sigma)^{*}(u)\right)$ is $\mathbb{C}$-algebraic, the map $f$ being regular. Thus, the cohomology class $u$ is blow- $\mathbb{C}$-algebraic, as asserted.

Example 5.1 should be compared with the following:

Example 5.10 For any positive integer $n$,

$$
\bar{H}_{\mathbb{C} \text {-alg }}^{\text {even }}\left(\mathbb{T}^{n} ; \mathbb{Z}\right)=H^{\text {even }}\left(\mathbb{T}^{n} ; \mathbb{Z}\right) .
$$

Obviously, $\bar{H}_{\mathbb{C} \text {-alg }}^{0}\left(\mathbb{T}^{n} ; \mathbb{Z}\right)=H^{0}\left(\mathbb{T}^{n} ; \mathbb{Z}\right)$. For any positive integer $k$, the cohomology group $H^{2 k}\left(\mathbb{T}^{n} ; \mathbb{Z}\right)$ is generated by cohomology classes of the form $[Y]^{\mathbb{T}^{n}}$, where $Y$ is a nonsingular codimension $2 k$ Zariski closed subvariety of $\mathbb{T}^{n}$ with trivial and oriented normal bundle. By [20, Theorem 2.4], there exists a regulous map $f: \mathbb{T}^{n} \rightarrow \mathbb{S}^{2 k}$ with $[Y]^{\mathbb{T}^{n}}=f^{*}\left(s_{2 k}\right)$, where $s_{2 k}$ is a generator of $H^{2 k}\left(\mathbb{S}^{2 k} ; \mathbb{Z}\right) \cong \mathbb{Z}$. Since the cohomology class $s_{2 k}$ is $\mathbb{C}$-algebraic, it follows from Theorem 5.8 that the cohomology class $[Y]^{\mathbb{T}^{n}}$ is blow- $\mathbb{C}$-algebraic. Consequently, $\bar{H}_{\mathbb{C} \text {-alg }}^{2 k}\left(\mathbb{T}^{n} ; \mathbb{Z}\right)=H^{2 k}\left(\mathbb{T}^{n} ; \mathbb{Z}\right)$, as asserted. 
It seems difficult to find precise relationships between $\bar{H}_{\mathbb{C} \text {-alg }}^{\text {even }}(-; \mathbb{Z})$ and $H^{\text {even }}(-; \mathbb{Z})$.

Example 5.11 Let $n$ and $k$ be integers satisfying $0<2 k<n$. According to Example 3.4, there exists a nonsingular affine real algebraic variety $X$ which is diffeomorphic to $\mathbb{T}^{n}$ and satisfies

$$
H_{\mathrm{alg}}^{2 k}(X ; \mathbb{Z} \backslash 2) \neq H^{2 k}(X ; \mathbb{Z} \backslash 2) .
$$

It follows from Proposition 5.12 below that

$$
\bar{H}_{\mathbb{C}-\text { alg }}^{2 k}(X ; \mathbb{Z}) \neq H^{2 k}(X ; \mathbb{Z}),
$$

the reduction modulo 2 homomorphism $H^{2 k}(X ; \mathbb{Z}) \rightarrow H^{2 k}(X ; \mathbb{Z} \backslash 2)$ being surjective.

Proposition 5.12 If $X$ is a compact nonsingular affine real algebraic variety, then

$$
\varrho\left(\bar{H}_{\mathbb{C}-\mathrm{alg}}^{2 k}(X ; \mathbb{Z})\right) \subseteq H_{\mathrm{alg}}^{2 k}(X ; \mathbb{Z} \backslash 2)
$$

for every $k \geq 0$, where $\varrho: H^{2 k}(-; \mathbb{Z}) \rightarrow H^{2 k}(-; \mathbb{Z} \backslash 2)$ is the reduction modulo 2 homomorphism.

Proof It is convenient first to prove a weaker assertion,

$$
\varrho\left(H_{\mathbb{C}-\text { alg }}^{2 k}(X ; \mathbb{Z})\right) \subseteq H_{\text {alg }}^{2 k}(X ; \mathbb{Z} \backslash 2) .
$$

To this end let $(V, \varepsilon)$ be a nonsingular projective complexification of $X$. Identifying $\mathbb{C}$ with $\mathbb{R}^{2}$, one can regard $V(\mathbb{C})$ as a real algebraic variety, denoted $V(\mathbb{C})_{\mathbb{R}}$, cf. [5, Proposition 3.4.6]. The variety $V(\mathbb{C})_{\mathbb{R}}$ is nonsingular and affine. By construction, $V(\mathbb{C})$ and $V(\mathbb{C})_{\mathbb{R}}$ coincide as topological spaces with the Euclidean topology. In particular, the following diagram is commutative:

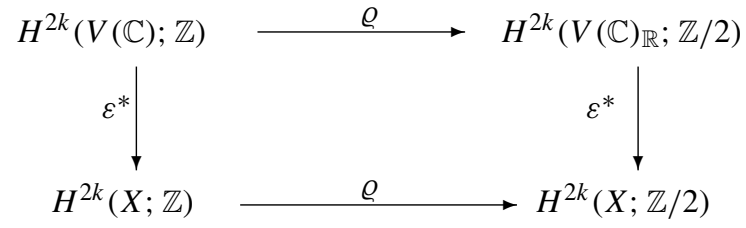

Since $\varepsilon^{*}\left(H_{\mathrm{alg}}^{2 k}(V(\mathbb{C}) ; \mathbb{Z})\right)=H_{\mathbb{C}-\mathrm{alg}}^{2 k}(X ; \mathbb{Z})$, one gets

(i)

$$
\varrho\left(H_{\mathbb{C}-\text { alg }}^{2 k}(X ; \mathbb{Z})\right)=\varepsilon^{*}\left(\varrho\left(H_{\text {alg }}^{2 k}(V(\mathbb{C}) ; \mathbb{Z})\right)\right)
$$

By construction,

(ii)

$$
\varrho\left(H_{\text {alg }}^{2 k}(V(\mathbb{C}) ; \mathbb{Z})\right) \subseteq H_{\text {alg }}^{2 k}\left(V(\mathbb{C})_{\mathbb{R}} ; \mathbb{Z} \backslash 2\right)
$$

Furthermore,

(iii)

$$
\varepsilon^{*}\left(H_{\mathrm{alg}}^{2 k}\left(V(\mathbb{C})_{\mathbb{R}} ; \mathbb{Z} \backslash 2\right)\right) \subseteq H_{\mathrm{alg}}^{2 k}(X ; \mathbb{Z} \backslash 2),
$$

the map $\varepsilon: X \rightarrow V(\mathbb{C})_{\mathbb{R}}$ being regular. The assertion follows by combining (1), (1) and (1). 
Now the proof can easily be completed. Let $u$ be a cohomology class in $\bar{H}_{\mathbb{C} \text {-alg }}^{2 k}(X ; \mathbb{Z})$ and let $\pi: X^{\prime} \rightarrow X$ be a multiblowup such that $\pi^{*}(u)$ is in $H_{\mathbb{C} \text {-alg }}^{2 k}\left(X^{\prime} ; \mathbb{Z}\right)$. According to the assertion proved above (applied to $X^{\prime}$ ), the cohomology class $\varrho\left(\pi^{*}(u)\right.$ ) is in $H_{\mathrm{alg}}^{2 k}\left(X^{\prime} ; \mathbb{Z} \backslash 2\right)$. Since the diagram

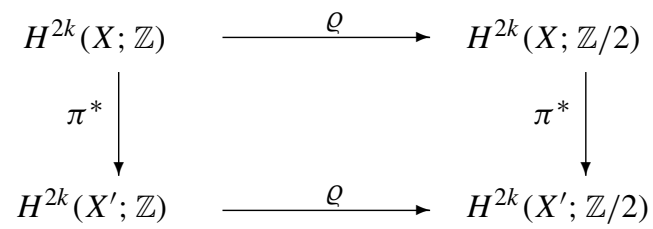

is commutative, the cohomology class $\pi^{*}(\varrho(u))$ is in $H_{\mathrm{alg}}^{2 k}\left(X^{\prime} ; \mathbb{Z} \backslash 2\right)$. Hence, according to Proposition 3.1, the cohomology class $\varrho(u)$ is in $H_{\mathrm{alg}}^{2 k}(X ; \mathbb{Z} \backslash 2)$, as required.

Open Access This article is distributed under the terms of the Creative Commons Attribution License which permits any use, distribution, and reproduction in any medium, provided the original author(s) and the source are credited.

\section{References}

1. Akbulut, S., King, H.: Submanifolds and homology of nonsingular real algebraic varieties. Amer. J. Math. 107, 45-83 (1985)

2. Benedetti, R., Tognoli, A.: On real algebraic vector bundles, Bull. Sci. Math. (2) 104 89-112 (1980)

3. Benedetti, R., Tognoli, A.: Remarks and counterexamples in the theory of real algebraic vector bundles and cycles, Géométrie algébrique réelle et formes quadratiques. Lecture Notes in Mathematics 959, pp. 198-211. Springer, Heidelberg (1982)

4. Bochnak, J., Buchner, M., Kucharz, W.: Vector bundles over real algebraic varietiesK-Theory 3 pp. 271-298 (1989). Erratum: $\mathcal{K}$-Theory 4 pp. 103 (1990)

5. Bochnak, J., Coste, M., Roy, M.-F.: Real Algebraic geometry, Ergeb. der Math. und ihrer Grenzgeb. Folge 3, vol. 36, Springer, Heidelberg (1998)

6. Bochnak, J., Kucharz, W.: Realization of homotopy classes by algebraic mappings. J. Reine Angew. Math. 377, 159-169 (1987)

7. Bochnak, J., Kucharz, W.: $\mathcal{K}$-Theory of real algebraic surfaces and threefolds. Math. Proc. Cambridge Philos. Soc. 106, 471-480 (1989)

8. Bochnak, J., Kucharz, W.: On homology classes represented by real algebraic varieties, Singularities Symposium (Kraków, : Banach Center Publ. 44. Warsaw 1998, 21-35 (1996)

9. Bochnak, J., Kucharz, W.: Real algebraic morphisms represent few homotopy classes. Math. Ann. 337, 909-921 (2007)

10. Borel et, A., Haefliger, A.: La classe d'homologie fondamentale d'un espace analytique, Bull. Soc. Math. France 89 pp. 461-513 (1961)

11. Coste, M., Diop, M. M.: Real algebraic 1-cocycles are nash coboundaries, Boll. Un. Mat. Ital. A (7) 6(2), 249-254 (1992)

12. Fichou, G., Huisman, J., Mangolte, F., Monnier, J.-Ph.: Fonctions régulues, arXiv:1112.3800v2 [math.AG] (2012)

13. Fulton, W.: Intersection theory, Ergeb. der Math. und ihrer Grenzgeb. Folge 3, vol. 2, Springer, Heidelberg (1984)

14. Hironaka, H.: Resolution of singularities of an algebraic variety over a field of characteristic zero. Ann. Math. 79, 109-326 (1964)

15. Hirsch, M.W.: Differential topology, graduate texts in mathematics 33, Springer, Heidelberg (1994) 
16. Huisman, J.: A real algebraic vector bundle is strongly algebraic whenever its total space is affine, Contemp. Math., 182, Amer. Math. Soc., Providence, RI. pp. 117-119 (1995)

17. Huisman, J.: Correction to "A real algebraic vector bundle is strongly algebraic whenever its total space is affine”, Contemp. Math., 253, Amer. Math. Soc., Providence, RI. p. 179 (2000)

18. Kollár, J.: Lectures on resolution of singularities, Ann. of Math. Studies 166, Princeton University Press, Princeton, NJ (2007)

19. Kollár, J.: Continuous rational functions on real and $p$-adic varieties, arXiv:1101.3737v1[math.AG] (2011)

20. Kucharz, W.: Rational maps in real algebraic geometry. Adv. Geom. 9, 517-539 (2009)

21. Milnor, J.W., Stasheff, J.D.: Characteristic classes, Ann of Math. Studies 76, Princeton University Press, Princeton, NJ (1974)

22. Tognoli, A.: Approximation theorems in real analytic and algebraic geometry, Lectures in real geometry (Madrid, 1996), de Gruyter Exp. Math., 23, pp. 113-166. Berlin, (1996) 